\title{
The Best Prediction Model for Trauma Outcomes of the Current Korean Population: a Comparative Study of Three Injury Severity Scoring Systems
}

\author{
Kyoungwon Jung, M.D., Ph.D. ${ }^{1}$, John Cook-Jong Lee, M.D., Ph.D. ${ }^{1}$, Rae Woong Park, M.D., Ph.D. ${ }^{2}$, \\ Dukyong Yoon, M.D., Ph.D. ${ }^{2}$, Sungjae Jung, B.S. ${ }^{2}$, Younghwan Kim, M.D. ${ }^{1}$, Jonghwan Moon, M.D. ${ }^{1}$, \\ Yo Huh, M.D. ${ }^{1}$, and Junsik Kwon, M.D. ${ }^{1}$
}

${ }^{1}$ Division of Trauma Surgery, Department of Surgery, ${ }^{2}$ Department of Biomedical Informatics, Ajou University School of Medicine, Suwon, Korea

\begin{abstract}
Background: Injury severity scoring systems that quantify and predict trauma outcomes have not been established in Korea. This study was designed to determine the best system for use in the Korean trauma population.

Methods: We collected and analyzed the data from trauma patients admitted to our institution from January 2010 to December 2014. Injury Severity Score (ISS), Revised Trauma Score (RTS), and Trauma and Injury Severity Score (TRISS) were calculated based on the data from the enrolled patients. Area under the receiver operating characteristic (ROC) curve (AUC) for the prediction ability of each scoring system was obtained, and a pairwise comparison of ROC curves was performed. Additionally, the cut-off values were estimated to predict mortality, and the corresponding accuracy, positive predictive value, and negative predictive value were obtained. Results: A total of 7,120 trauma patients ( 6,668 blunt and 452 penetrating injuries) were enrolled in this study. The AUCs of ISS, RTS, and TRISS were $0.866,0.894$, and 0.942 , respectively, and the prediction ability of the TRISS was significantly better than the others ( $p$ $<0.001$, respectively). The cut-off value of the TRISS was 0.9082 , with a sensitivity of $81.9 \%$ and specificity of $92.0 \%$; mortality was predicted with an accuracy of $91.2 \%$; its positive predictive value was the highest at $46.8 \%$.

Conclusions: The results of our study were based on the data from one institution and suggest that the TRISS is the best prediction model of trauma outcomes in the current Korean population. Further study is needed with more data from multiple centers in Korea.
\end{abstract}

Key Words: Korea; injury severity score; mortality; prediction; trauma centers; outcomes.

\section{Introduction}

Trauma is a worldwide health problem, but its burden is skewed more heavily in developing or underdeveloped countries.[1] Disparity in regard to trauma in developing countries is due to a variety of factors, including lack of approved trauma centers, lack of highly trained medical professionals, and insufficient experience with trauma assessment tools. The prognosis of trauma patients differs greatly among countries with and without a well-organized trauma manage-

Received on May 31, 2016 Revised on July 25, 2016 Accepted on July 29, 2016

Correspondence to: Junsik Kwon, Division of Trauma Surgery, Department of Surgery, Ajou University School of Medicine, 206 World cup-ro, Yeoungtong-gu, Suwon 16499, Korea

Tel: +82-31-219-7491, Fax: +82-31-219-7765

E-mail: aquaeste|@gmail.com

*No potential conflict of interest relevant to this article was reported. ment system; a study that compared the mortality rate of patients with an Injury Severity Score (ISS) $\geq 15$ in lowincome versus high-income countries found that the mortality rate was approximately six-fold higher in low-income countries.[2] Such an enormous difference impedes the global standardization of predicting the prognosis of trauma patients and establishment of criteria to fairly as- 
sess a country's trauma system.[3] Korea is a socioeconomically developed country that recently made progress in its health care system. However, a recent survey[4] showed a mortality rate of $35.2 \%$ among trauma patients whose deaths were preventable; thus, in terms of trauma management, Korea is similar to a developing country. Korea is working on establishing an organized trauma system to decrease the morality rate of trauma patients whose deaths are preventable to $20 \%$ or lower by 2020 . An injury severity scoring system (ISSS) will be created to quantify the severity of trauma to compare and predict outcomes across different trauma centers and to quantitatively determine whether Korea's trauma system should be improved.[5] Since 1971, when an ISSS called the Abbreviated Injury Scale (AIS)[6] was developed in the United States, several scoring systems have been developed. In Korea, traumatology is in the beginning stage of development compared to other advanced countries. A few studies have been conducted on an ISSS in the Korean population. However, these studies included a small sample size[7] or a survey of motor vehicle accident victims.[8] It would be of great significance to evaluate whether representative ISSSs can be preliminarily used in Korea before an appropriate scoring system is created for the Korean population. Therefore, we assessed three representative ISSSs, i.e., ISS, Revised Trauma Score (RTS), and Trauma and Injury Severity Score (TRISS), in terms of ability to predict the outcome of trauma treatment.

\section{Materials and Methods}

\section{1) Study population}

The data of patients aged $\geq 15$ years who were treated as inpatients at the author's institution between January 2010 and December 2014 were analyzed. The institution is a main tertiary referral center in Korea where approximately 17,000 trauma patients / year visit the emergency department. Of those, $>2,000$ patients/year are admitted and treated by the department in charge of trauma, and the rate of severe trauma patients with an ISS score $\geq 15$ is $23 \%$ on average. Since 2013 , the hospital has collaborated with government-run regional trauma centers, and it operates a trauma center with 20 intensive care unit (ICU) beds.

\section{2) ISSSS and the inclusion criteria}

For each patient, the ISS, RTS, and TRISS were computed. Patients were included if the AIS coding during the resuscitation process was available in the medical record and if their initial level of consciousness was included in the medical record from the transferring hospital or ambulance service record in the case that the Glasgow Coma Scale (GCS) could not be assessed due to intubation during transfer. The following patients were excluded: those in which the AIS code was missing, as autopsies are rarely performed in Korea; patients whose ISS, RTS, and TRISS could not be computed because the GCS score, initial vital signs, or other clinical data were inaccurate; those who were dead on arrival; patients who died in the emergency department; and those whose initial level of consciousness could not be confirmed.

Physiological parameters used in the RTS were assessed using ambulance service records, medical records from the transferring hospitals, etc. in order to identify the initial values. The AIS used for the ISS was based on the AIS $2005^{\circ}$ Update 2008 (Association for the Advancement of Automotive Medicine, Barrington, IL, USA). Three trauma coordinators performed the coding, and six trauma panel members (three general surgeons, one orthopedist, one neurosurgeon, and one radiologist) reviewed and finalized it. The TRISS was computed using the Major Trauma Outcome Study (MTOS)-derived coefficient revised in 1995.[9]

\section{3) Statistical analysis}

To examine the predictive ability of each ISSS for death, areas under the receiver operating characteristic (ROC) curve (areas under the receiver curve [AUC]) were computed and statistically compared. All comparisons were analyzed using the $\mathrm{R}$ package (version 3.1.2, $\mathrm{R}$ 
Foundation for Statistical Computing, Vienna, Austria); specifically, the AUC and 95\% confidence interval were computed, and the pROC package was used to compare the three scoring systems.[10] A p value $<0.05$ was con-

Table 1. Characteristics of the study population

\begin{tabular}{|c|c|}
\hline Variable & Total study cohort \\
\hline $\mathrm{N}$ & 7,120 \\
\hline Age (years) & $48.9 \pm 19.3$ \\
\hline \multicolumn{2}{|l|}{ Sex } \\
\hline Male & $4,826(67.8)$ \\
\hline Female & 2,294 (32.2) \\
\hline \multicolumn{2}{|l|}{ Transfer route } \\
\hline Direct & $3,718(52.2)$ \\
\hline From another hospital & $3,402(47.8)$ \\
\hline \multicolumn{2}{|l|}{ Mechanism } \\
\hline Blunt & $6668(93.7)$ \\
\hline Traffic accident & $2679(40.2)$ \\
\hline Fall & $1395(20.9)$ \\
\hline Ground fall ${ }^{*}$ & $1312(19.7)$ \\
\hline Violence & $856(12.8)$ \\
\hline Unspecified/not elsewhere classifiable & $426(6.4)$ \\
\hline Penetrating & $452(6.3)$ \\
\hline Respiratory rate (beats per min) & $16.2 \pm 5.0$ \\
\hline \multicolumn{2}{|l|}{ Respiratory rate } \\
\hline $0-5$ breaths per min & $192(2.7)$ \\
\hline$>29$ breaths per min & $150(2.1)$ \\
\hline 10-29 breaths per min & 6,778 (95.2) \\
\hline Systolic blood pressure (mmHg) & $126.9 \pm 32.4$ \\
\hline \multicolumn{2}{|l|}{ Systolic blood pressure } \\
\hline$<90 \mathrm{mmHg}$ & $485(6.8)$ \\
\hline$\geq 90 \mathrm{mmHg}$ & 6,635 (93.2) \\
\hline Glasgow Coma Scale score & $13.7 \pm 3.2$ \\
\hline \multicolumn{2}{|l|}{ Glasgow Coma Scale } \\
\hline 3 & $346(4.9)$ \\
\hline $4-5$ & $129(1.8)$ \\
\hline $6-8$ & $246(3.5)$ \\
\hline $9-12$ & $310(4.4)$ \\
\hline 13-15 & $6,089(85.5)$ \\
\hline Injury Severity Score & $11.6 \pm 10.4$ \\
\hline Revised Trauma Score & $7.3376 \pm 1.4959$ \\
\hline Trauma and Injury Severity Score & $0.9209 \pm 0.1944$ \\
\hline
\end{tabular}

Values are presented as mean \pm SD or $n(\%)$.

*Fall from standing height. sidered statistically significant.

Additionally, the cut-off values associated with the highest sensitivity and specificity values for each scoring system were estimated to predict mortality, and the corresponding accuracy, positive predictive value, and negative predictive value were obtained. The Youden Index[11] was used to compute the cut-off values.

\section{4) Ethics statement}

This study was approved by the Institutional Review Board of Ajou University Hospital (AJIRB-MEDMDB-16-013). Informed consent was waived by the board due to the observational nature of the study.

\section{Results}

During the study period, 9,142 patients received inpatient treatment from the Department of Trauma Surgery; of those, 7,120 (6,668 [93.7\%] with blunt injuries and 452 [6.3\%] with penetrating injuries) met the inclusion criteria and were analyzed. The majority of patients were men $(4,826 ; 67.8 \%)$, and mean patient age was $48.85 \pm 19.25$ years. Overall, 3,402 (47.8\%) patients were transferred from another hospital. The respiratory rate per minute was $\geq 30$ breaths or $\leq 5$ breaths in 342 (2.8\%) patients. The systolic blood pressure (SBP) was $<90 \mathrm{mmHg}$ in 485 (6.8\%) patients. The GCS score was $\leq 8$ in $721(10.1 \%)$ patients (mean GCS score, $13.7 \pm$ 3.2). The most common type of injury was blunt (6668), and traffic accidents were most prevalent, accounting for $40.2 \%$ of submitted patients (Table 1). Five hundred sixty-two patients died, with an overall mortality rate of

Table 2. Areas under the ROC curve for the predictive ability of each injury severity scoring system

\begin{tabular}{lcc}
\hline Scoring system & AUC & $95 \% \mathrm{Cl}$ \\
\hline ISS & 0.866 & $0.851-0.881$ \\
RTS & 0.894 & $0.877-0.910$ \\
TRISS & 0.942 & $0.932-0.953$ \\
\hline
\end{tabular}

ROC: receiver operating characteristic; AUC: area under the receiver operating characteristic curve; Cl: confidence interval; ISS: Injury Severity Score; RTS: Revised Trauma Score; TRISS: Trauma and Injury Severity Score. 
$7.9 \%$. The mean values of ISS, RTS, and TRISS (survival probability) were $11.6 \pm 10.4,7.34 \pm 1.53$, and $0.92 \pm 0.19$, respectively. The AUCs for the predictive ability of the ISS, RTS, and TRISS were 0.866, 0.894, and 0.942, respectively (Table 2). The predictive ability of the TRISS was significantly better than those of the ISS $(\mathrm{p}<0.001)$ and RTS ( $<<0.001$; Table 3, Fig. 1).

The cut-off values of each scoring system are shown

Table 3. Comparison of the ISS, RTS, and TRISS in terms of predicting mortality

\begin{tabular}{lcr}
\hline & AUC & p-value \\
\hline ISS vs. RTS & 0.866 vs. 0.894 & 0.002 \\
ISS vs. TRISS & 0.866 vs. 0.942 & $<0.001$ \\
RTS vs. TRISS & 0.866 vs. 0.942 & $<0.001$ \\
\hline
\end{tabular}

ISS: Injury Severity Score; RTS: Revised Trauma Score; TRISS: Trauma and Injury Severity Score; AUC: area under the receiver operating characteristic curve.

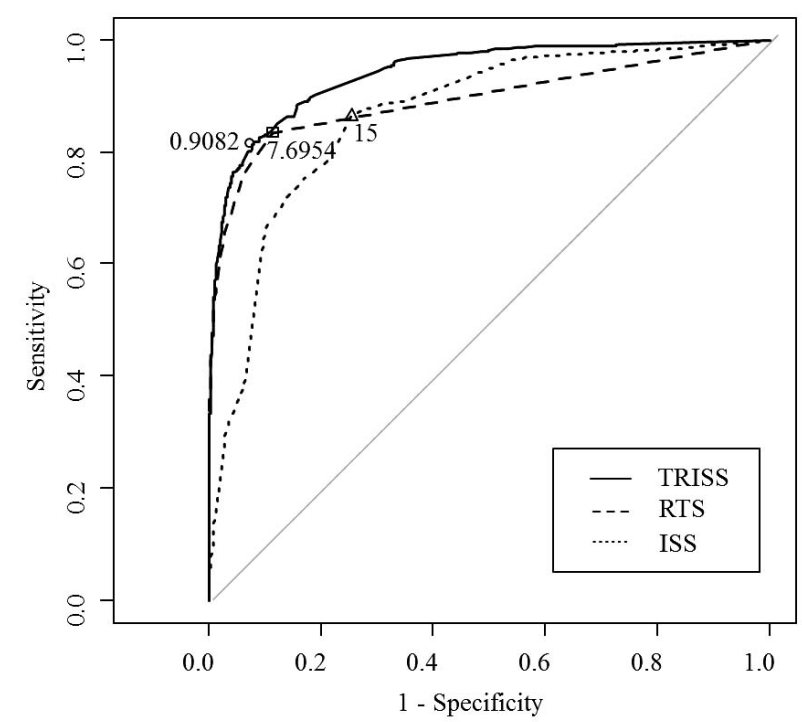

Fig. 1. Receiver operating characteristic curves of Injury Severity Score (ISS), Revised Trauma Score (RTS), and Trauma and Injury Severity Score (TRISS) for predicting mortality in the study population. in Table 4. The cut-off value of the ISS was 15 , with a sensitivity of $86.7 \%$ and specificity of $74.6 \%$; mortality was predicted with an accuracy of $75.5 \%$. The positive predictive value was low $(22.6 \%)$, whereas the negative predictive value was very high $(98.5 \%)$. The cut-off value of the RTS was 7.6954, with a sensitivity of $83.5 \%$ and specificity of $88.6 \%$; mortality was predicted with an accuracy of $88.2 \%$. The positive predictive value was $38.5 \%$, which was higher than that of the ISS, and the negative predictive value was $98.4 \%$, which was similar to that of the ISS. The cut-off value of the TRISS was 0.9082 , with a sensitivity of $81.9 \%$ and specificity of $92.0 \%$; mortality was predicted with an accuracy of $91.2 \%$. Overall, the TRISS had the best results among the three ISSSs; its positive predictive value was the highest at $46.8 \%$.

\section{Discussion}

It is critical to establish an ideal trauma scoring system due to its many uses, including predicting the prognosis of trauma patients, comparing treatment methods, using it as a triage tool during transport to a hospital or during transfer between hospitals, using it as a tool for quality management of trauma centers, using it as a preventive program, and using it as a research tool in trauma studies.[12] We used scoring systems that have been developed and widely utilized in countries in which traumatology has progressed, including Korea, to investigate the benefits and limitations of ISSSs. More blunt injuries than penetrating injuries occur in Korea compared to the United States because the use of guns is restricted. Thus, it is important to evaluate the applicability and outcome prediction ability of existing ISSSs primarily developed

Table 4. Cut-off value of each scoring system at the maximum sensitivity and specificity and the accuracy, positive, and negative predictive values of the receiver operating characteristic curve

\begin{tabular}{lcccccc}
\hline Scoring system & Cut-off value & Sensitivity (\%) & Specificity (\%) & Accuracy (\%) & PPV (\%) & NPV (\%) \\
\hline ISS & 15 & 86.7 & 74.6 & 75.5 & 22.6 & 98.5 \\
RTS & 7.6954 & 83.4 & 88.6 & 88.2 & 38.5 & 98.4 \\
TRISS & 0.9082 & 81.9 & 92.1 & 91.2 & 46.8 & 98.3 \\
\hline
\end{tabular}

PPV: positive predictive value; NPV: negative predictive value; ISS: Injury Severity Score; RTS: Revised Trauma Score; TRISS: Trauma and Injury Severity Score. 
and widely used in the United States in the Korean patient population. To establish a countrywide trauma system, Korea has established $>10$ regional trauma centers and supported them with tax revenues, but it does not yet have a program or specific plans for quality improvement of the centers' operation. The Korean government has set a goal to reduce the mortality rate of preventable deaths to $<20 \%$ by 2020 . However, the current mortality rate of preventable deaths, which was the baseline used to set the goal, was determined in a subjective panel review; thus, the objectivity has been debated. Existing research and research efforts are lacking on the topic of ISSSs, so this debate continues. Currently, a trauma database is being established at medical facilities selected as regional trauma centers, but it is not available to general researchers because the reliability of the data is low; furthermore, no attempt has been made to establish Korean norms, such as the MTOS in the United States. Therefore, the present study's findings, which were based on data accumulated from the time that the first trauma department in Korea began to operate, would be helpful in establishing the foundation needed to operate these recently developed regional trauma centers and trauma system.

The ISS was first suggested by Baker et al.[13] in 1974, and it is a method for classifying trauma patients based on anatomic measures. It was founded on the AIS, which was created with the goal of categorizing the severity and type of injuries caused by a car crash, and it is commonly used as an anatomical indicator to classify injury severity. The ISS is intuitive in severity evaluation and mortality prediction, and it is well associated with the mortality rate. However, it is accurately calculated only in the presence of objective data such as accurate test findings, surgery or autopsy results, etc.; thus, it has a limitation in assessing the severity of an emergency patient at an early stage.[14] Additionally, the ISS is limited in that severity is not accurately reflected in some trauma cases, as it does not indicate more than two overlapping injuries in the same area. Thus, some researchers have suggested the New Injury Severity Score (NISS) that sums the three highest AIS scores, regardless of the physical area.
[15] However, like the ISS, it does not include physiological indices, making it difficult to accurately predict the prognosis. A few studies have reported that the NISS did not differ from or was even worse than the ISS for predicting mortality, admission to the ICU, and length of hospital stay,[16,17] and it is not widely used. In the present study, we confirmed the usefulness of the ISS in the Korean trauma patient population, and we found that its predictive ability was not poor. Particularly, the cutoff value of 15 confirmed the conventional criterion of using 15 points to define a severe trauma patient.[18]

The RTS was introduced in the early 1980s, and it is the most widely used physiologic scoring system to classify trauma patients.[17] It uses three physiological parameters, the GCS, SBP, and respiratory rate (RR), to score injuries, with a minimum score of 0 and a maximum score of 7.8408. A lower score indicates a more severe injury; thus, a lower survival rate is estimated. The RTS is excellent for predicting the mortality rate, it is suitable to use before the trauma patient is transported to a hospital because it is based on indices easily obtained in the field, and it is widely used. However, the GCS and RR cannot be accurately assessed in intubated or sedated patients. Moreover, in many developing countries or areas where a trauma system is not well established, prehospital records are incomplete or difficult to rely on, and many patients go to their local hospital first before they are transferred to a trauma center; hence, there is a limitation in obtaining physiological parameters early. Yet, some studies have reported significant results from routinely using the data measured immediately after patients are transferred to the hospital to score the injury without adjusting for the time passed after the injury.[14,19-22] We made great efforts to obtain ambulance service records and the medical records from transferring hospitals and reviewed them meticulously to determine the earliest physiological values; the cases for which we were not able to obtain accurate values were excluded. We believe that such efforts resulted in an AUC value comparable to that in previous research.[23]

The TRISS is a combined scoring system that was 
developed in 1987,[24] and it is based on the ISS as an anatomic component, RTS as a physiological component, and patient age as a comorbid component, resulting in a combined index for injury that complements each component. This scoring system is the most representative method to compute the probability of survival of a trauma patient, and it retrospectively applies a logistic regression model to compute the probability using the following equation; the solution values of which range from $0-1$.

$$
\begin{aligned}
& \text { Probability of Survival }=1 \div\left(1+\mathrm{e}^{-\mathrm{b}}\right) \\
& \mathrm{b}=\alpha+\beta_{\mathrm{AGE}} \times \mathrm{AGE}+\beta_{\mathrm{RR}} \times \mathrm{RR}+\beta_{\mathrm{SBP}} \times \mathrm{SBP}+\beta_{\mathrm{GCS}} \times \\
& \mathrm{GCS}+\beta_{\mathrm{ISS}} \times \mathrm{ISS}
\end{aligned}
$$

In the equation, $\alpha, \beta_{\mathrm{AGE}}, \beta_{\mathrm{RR},}, \beta_{\mathrm{SBP}}, \beta_{\mathrm{GCS}}$, and $\beta_{\mathrm{ISS}}$ are constants defined differently for patients with blunt trauma and those with penetrating trauma. In our study, we used the MTOS-derived coefficients revised in 1995.[25]

We computed the ROC curves of the ISS, RTS, and TRISS; estimated the respective AUCs; and statistically compared them to determine the ability of each scoring system to predict the mortality rate, which is the most important outcome measure of trauma. Generally, when an AUC value from ROC analysis is 0.9 or higher, the method is considered highly accurate.[26] In our study, the AUC of the TRISS was 0.942 , suggesting that it is a very accurate method for predicting the mortality rate, and it was statistically better compared to the ISS and RTS. It has been consistently reported that the TRISS has superior results for predicting the mortality rate of trauma patients in developed countries compared to other scoring systems.[27-29] Thus, the TRISS may play a very important role in predicting mortality in a developing country such as Korea where the trauma patient population is different from that of a developed country and no trauma system has yet been established.

We believe that the respective cut-off value of the ISSSs is significant. In particular, the cut-off value of 15 for the ISS is consistent with the convention of classifying a severe patient with a high risk of death with a score $\geq 15$. If the medical staff that specializes in trauma correctly use the AIS codes and apply the ISS according to its principles, the ISS can be a very useful tool for assessing the trauma patient population in Korea. The cutoff value for the TRISS was 0.9082 (90.82\% survival probability). To effectively utilize these values as a tool for assessing the objective probability of death, both the positive predictive value and negative predictive value must be high; however, in the present study, the positive predictive value was $46.8 \%$ and the negative predictive value was $98.3 \%$. Cayten et al.[27] reported that the positive predictive value of the TRISS for predicting death was $21.5 \%$, and it was only $16 \%$ in patients who experienced a fall from standing height. Karmy-Jones et al.[30] reported that the positive predictive value of the TRISS was only $16.1 \%$. Given these previous findings, our finding of $46.8 \%$ is comparable.

There are a few limitations in the study. First, the number of patients transferred from another hospital was as high as 3,400 , which was almost half of the total sample. This may raise an issue regarding the measurement of the physiological parameters, which in turn affects the reliability of the RTS and TRISS results. To rectify this problem, we reviewed the prehospital records and consequently obtained results comparable to previously reported findings. Second, although the TRISS is a populationbased scoring system, we applied constants that were computed based on the patient population of another region comparable to the Korean patients, and the study was conducted at a single trauma center. Recently, an epidemiological study conducted by a trauma center in Japan reported a significant difference between the coefficients computed from the Japanese data and the MTOS coefficients, and the authors argued that patient outcome in a certain area should be assessed using the TRISS coefficients, which are modified according to the area. [31] Considering this Japanese study, the present study's findings cannot be generalized to the Korean trauma population. Therefore, new TRISS coefficients should be computed in a large-scale multi-center study with Korean trauma patients who have unique characteristics, or an outcome prediction model for Korean trauma patients 
should be developed.

When establishing a trauma system, a trauma scoring system is very important. To create a scoring system for the patient population in a developed country that can be used worldwide, the scoring system first has to be evaluated in a developing country that is different from a developed country in terms of its socioeconomic state and maturity of its trauma system. Thus, we used existing representative trauma scoring systems, i.e., the ISS, RTS, and TRISS, and compared the estimated mortality rates of trauma patients in a developing country. The results showed that these ISSSs can be very useful in assessing trauma patients in Korea, which is a developing country in terms of its trauma system, although the patient population is different from that of the country where the scoring systems were originally developed. Specifically, among the three ISSSs, the TRISS may be the most effective. However, additional research is needed to develop coefficients specific to Korea considering that the TRISS is a population-based scoring system.

\section{Acknowledgements}

Thanks: The authors would like to thank trauma program manager Jiyoung Kim, trauma coordinators Juryang Kim and Hyoju Kim for collecting and cleansing of data and AIS coding.

Grant support for the research: This work was supported by the new faculty research fund of Ajou University School of Medicine.

\section{ORCID}

$\begin{array}{ll}\text { Kyoungwon Jung } & \text { http://orcid.org/0000-0001-7895-0362 } \\ \text { John Cook-Jong Lee } & \text { http://orcid.org/0000-0002-5468-3340 } \\ \text { Rae Woong Park } & \text { http://orcid.org/0000-0003-4989-3287 } \\ \text { Dukyong Yoon } & \text { http://orcid.org/0000-0003-1635-8376 } \\ \text { Sungjae Jung } & \text { http://orcid.org/0000-0001-8266-0480 } \\ \text { Younghwan Kim } & \text { http://orcid.org/0000-0003-3836-8743 } \\ \text { Jonghwan Moon } & \text { http://orcid.org/0000-0002-4630-3301 } \\ \text { Yo Huh } & \text { http://orcid.org/0000-0002-1220-1534 } \\ \text { Junsik Kwon } & \text { http://orcid.org/0000-0003-3164-4995 }\end{array}$

\section{References}

1) Peden M, Mcgee KS, Sharma G: The injury chart book: a graphical overview of the global burden of injuries. Geneva: World Health Organization; 2002.

2) Mock CN, Adzotor KE, Conklin E, Denno DM, Jurkovich GJ: Trauma outcomes in the rural developing world: comparison with an urban level I trauma center. J Trauma 1993; 35: 518-23.

3) Trunkey DD: The medical world is flat too. World J Surg 2008; 32: 1583-604.

4) Kim H, Jung KY, Kim SP, Kim SH, Noh H, Jang HY, et al: Changes in preventable death rates and traumatic care systems in Korea. J Korean Soc Emerg Med 2012; 23: 189-97.

5) Wisner DH: History and current status of trauma scoring systems. Arch Surg 1992; 127: 111-7.

6) Rating the severity of tissue damage. I. The abbreviated scale. JAMA 1971; 215: 277-80.

7) Kim KH, Kim HY: Assessment of the usefulness of various trauma scoring systems on the prognosis of multiple-injury patients. J Korean Surg Soc 1998; 54(Suppl): 943-50.

8) Hwang JY, Lee KH, Shin HJ, Cha KC, Kim H, Hwang SO: Correlation analysis of trauma scoring system in predictive validity in motor vehicle accident. J Korean Soc Emerg Med 2011; 22: 329-34.

9) Champion HR, Sacco WJ, Copes WS, Gann DS, Gennarelli TA, Flanagan ME: A revision of the trauma score. J Trauma 1989; 29: 623-9.

10) Robin $X$, Turck N, Hainard A, Tiberti N, Lisacek F, Sanchez JC, et al: pROC: an open-source package for $\mathrm{R}$ and $\mathrm{S}+$ to analyze and compare ROC curves. BMC Bioinformatics 2011; 12: 77.

11) Schisterman EF, Perkins NJ, Liu A, Bondell H: Optimal cut-point and its corresponding Youden index to discriminate individuals using pooled blood samples. Epidemiology 2005; 16: 73-81.

12) Chawda MN, Hildebrand F, Pape HC, Giannoudis PV: Predicting outcome after multiple trauma: which scoring system? Injury 2004; 35: 347-58. 
13) Baker SP, O’Neill B, Haddon W Jr, Long WB: The injury severity score: a method for describing patients with multiple injuries and evaluating emergency care. J Trauma 1974; 14: 187-96.

14) Samuel JC, Akinkuotu A, Baloyi P, Villaveces A, Charles A, Lee CN, et al: Hospital-based injury data in Malawi: strategies for data collection and feasibility of trauma scoring tools. Trop Doct 2010; 40: 98-9.

15) Osler T, Baker SP, Long W: A modification of the injury severity score that both improves accuracy and simplifies scoring. J Trauma 1997; 43: 922-6; discussion 925-6.

16) Tamim H, Al Hazzouri AZ, Mahfoud Z, Atoui M, El-Chemaly S: The injury severity score or the new injury severity score for predicting mortality, intensive care unit admission and length of hospital stay: experience from a university hospital in a developing country. Injury 2008; 39: 115-20.

17) Tay SY, Sloan EP, Zun L, Zaret P: Comparison of the new injury severity score and the injury severity score. J Trauma 2004; 56: 162-4.

18) Becher RD, Meredith JW, Kilgo PD: Injury severity scoring and outcomes research. In: Trauma. 7th ed. Edited by Mattox KL, Moore EE, Feliciano DV: New York, McGraw-Hill. 2013, pp 77-90.

19) Ahun E, Köksal Ö, Sığırlı D, Torun G, Dönmez SS, Armağan E: Value of the Glasgow coma scale, age, and arterial blood pressure score for predicting the mortality of major trauma patients presenting to the emergency department. Ulus Travma Acil Cerrahi Derg 2014; 20: 241-7.

20) Haac B, Varela C, Geyer A, Cairns B, Charles A: The utility of the Kampala trauma score as a triage tool in a sub-Saharan African trauma cohort. World J Surg 2015; 39: 356-62.

21) Rastogi D, Meena S, Sharma V, Singh GK: Causality of injury and outcome in patients admitted in a major trauma center in North India. Int J Crit Illn Inj Sci 2014; 4: 298-302.

22) Weeks SR, Juillard CJ, Monono ME, Etoundi GA, Ngamby MK, Hyder AA, et al: Is the Kampala trauma score an effective predictor of mortality in lowresource settings? A comparison of multiple trauma severity scores. World J Surg 2014; 38: 1905-11.

23) Schluter PJ, Nathens A, Neal ML, Goble S, Cameron CM, Davey TM, et al: Trauma and Injury Severity Score (TRISS) coefficients 2009 revision. J Trauma 2010; 68: 761-70.

24) Boyd CR, Tolson MA, Copes WS: Evaluating trauma care: the TRISS method. Trauma Score and the Injury Severity Score. J Trauma 1987; 27: 370-8.

25) Champion HR, Sacco WJ, Copes WS: Injury severity scoring again. J Trauma 1995; 38: 94-5.

26) Altman D, Machin D, Bryant T, Gardner S: Statistics with confidence: confidence intervals and statistical guidelines. 2nd ed. Bristol, BMJ Books. 2000.

27) Cayten CG, Stahl WM, Murphy JG, Agarwal N, Byrne DW: Limitations of the TRISS method for interhospital comparisons: a multihospital study. J Trauma 1991; 31: 471-82; discussion 481-2.

28) Gabbe BJ, Cameron PA, Wolfe R: TRISS: does it get better than this? Acad Emerge Med 2004; 11: 181-6.

29) Glance LG, Osler T: Beyond the major trauma outcome study: benchmarking performance using a national contemporary, population-based trauma registry. J Trauma 2001; 51: 725-7.

30) Karmy-Jones R, Copes WS, Champion HR, Weigelt J, Shackford S, Lawnick M, et al: Results of a multiinstitutional outcome assessment: results of a structured peer review of TRISS-designated unexpected outcomes. J Trauma 1992; 32: 196-203.

31) Fujita T, Morimura N, Uchida Y, Kaneko I, Ikeda H, Sakamoto T: M-study from an urban trauma center in Tokyo. J Trauma 2010; 69: 934-7. 